\section{Relação entre índices fisiológicos aeróbios e desempenho em provas de curta e média duração em ciclistas de elite}

\section{Relationship between physiological indices and aerobic performance tests in short and medium term of elite cyclists}

\author{
Eduardo Bernardo Sangali ${ }^{1}$ \\ Eduardo Zapaterra Campos ${ }^{1}$ \\ Luigi Agostini Gobbo ${ }^{1}$ \\ Vitor Luiz de Andrade ${ }^{1}$ \\ Marcelo Papoti ${ }^{1}$ \\ Ismael Forte Freitas Júnior ${ }^{1}$ \\ Tiago Rezende Figueira ${ }^{2}$ \\ Pedro Balikian Junior ${ }^{1}$
}

Resumo - Poucos são os estudos que possibilitam verificar quais as respostas fisiológicas são associadas ao desempenho em uma amostra de ciclistas de elite nacional. Portanto, o objetivo do presente estudo foi determinar e relacionar diferentes índices fisiológicos aeróbios com o desempenho em testes contra relógio de 4 e $20 \mathrm{~km}$ em ciclistas de alto nível. A amostra foi composta por 14 ciclistas profissionais de elite nacional do sexo masculino $(28,5 \pm 4,7$ anos, $73,47 \pm 8,29 \mathrm{~kg}, 176 \pm 6,76 \mathrm{~cm})$, que realizaram um teste progressivo em laboratório para a determinação do consumo máximo de oxigênio $\left(\mathrm{VO}_{2} \max : 62,23 \pm 8,28\right.$ $\left.\mathrm{ml} \cdot \mathrm{kg} \cdot \mathrm{min}^{-1}\right)$, intensidade relativa ao $\mathrm{VO}_{2} \max \left(\mathrm{iVO}_{2} \max : 500,83 \pm 58,65 \mathrm{w}\right)$, economia de movimento (EM: 0,1166 $\pm 0,0362 \mathrm{ml} \cdot \mathrm{kg} \cdot \mathrm{min} \cdot \mathrm{w}^{-1}$ ) e $1^{\circ} \mathrm{e} 2^{\circ}$ limiares ventilatórios (LV1: $348,21 \pm 43,26 \mathrm{w} ;$ LV2: 417,86 $\pm 60,79 \mathrm{w}$, respectivamente). Também foram submetidos a duas provas de 4 e $20 \mathrm{~km}$ contra relógio. Para correlação entre os índices fisiológicos e desempenho, foi utilizado o coeficiente de correlação de Pearson $(p<0,05)$. Não foi encontrada correlação entre os índices fisiológicos ( $\mathrm{VO}_{2} \max$ absoluto e relativo, $\mathrm{iVO}_{2} \max$, EM, LV1 e LV2) e o desempenho de $4 \mathrm{~km}(r=0.38 ; 0.16 ;-0.33 ; 0.20 ;-0.50 ;-0.20$, respectivamente) e $20 \mathrm{~km}$ ( $r=0.24 ; 0.01 ;-0.13 ;-0.12 ;-0.48 ;-0.19$, respectivamente) contra relógio em atletas de alto nível. Estes resultados sugerem que tais variáveis não apresentam capacidade de explicar o desempenho em provas de contra relógio nas respectivas distâncias, provavelmente, devido à homogeneidade entre os sujeitos.

Palavras-chave: Avaliação de desempenho; Ciclismo; Consumo de Oxigênio; Limiar anaeróbio.

\begin{abstract}
Few studies allow us to verify which physiological responses are associated with performance in a national elite cycling group. Therefore, this study aimed to determine and correlate various physiological and aerobic indices with performance in 4 and $20 \mathrm{~km}$ time trials in high-level cyclists. The sample consisted of 14 male professional cyclists of the national elite group ( $28.5 \pm 4.7$ years old, $73.47 \pm 8.29 \mathrm{~kg}, 176 \pm 6.76 \mathrm{~cm})$, who performed a progressive test in laboratory to determine maximal oxygen consumption ( $\mathrm{VO}_{2}$ max: $62.23 \pm 8.28 \mathrm{ml} \cdot \mathrm{kg}$ 1•min-1), intensity relative to $\mathrm{VO}_{2} \max \left(i \mathrm{VO}_{2} \max : 500.83 \pm 58.65 \mathrm{w}\right.$ ), movement economy (EM: $0.1166 \pm 0.0362 \mathrm{ml} \bullet \mathrm{kg} \bullet \mathrm{min} \bullet \mathrm{w}-1$ ), and the first and second ventilatory threshold (LV1: $348.21 \pm 43.26 w$; LV2: $417.86 \pm 60.79 w$, respectively). They also performed two time trial performance tests of 4 and $20 \mathrm{~km}$. For the correlation between physiological indices and trial performance, Pearson correlation coefficient $(p<0.05)$ was used. No correlation was found between the physiological indices $\left(\mathrm{VO}_{2}\right.$ max absolute and relative, $i \mathrm{VO}_{2}$ max, EM, LV1 and $L V 2)$ and performance in $4 \mathrm{~km}(r=0.38 ; 0.16 ;-0.33 ; 0.20 ;-0.50 ;-0.20$, respectively $)$ and $20 \mathrm{~km}(r=0.24 ; 0.01 ;-0.13 ;-0.12 ;-0.48 ;-0.19$, respectively) time trial in high level athletes. These results suggest that these variables are not able to explain the performance in time trials in the respective lengths, probably due to the subjects' homogeneity.
\end{abstract}

Key words: Anaerobic Threshold; Bicycling; Employee performance appraisal; Oxygen consumption.
1 Universidade Estadual Paulista. Faculdade de Ciências e Tecnologia. Presidente Prudente, SP. Brasil.

2 Universidade Estadual de Campinas. Programa de Pós-Graduação em Clínica Médica. Campinas, SP. Brasil.

Recebido em 07/03/12 Revisado em 03/09/12 Aprovado em 09/11/12 


\section{INTRODUÇÃO}

Não encontramos na literatura estudos envolvendo ciclistas de elite, considerando os atletas melhores ranqueados pela Confederação Brasileira de Ciclismo (CBC), que apesar das dificuldades de se realizar experimentos bem controlados quanto à ausência de sobrecarga de treinamento, ou das frequentes competições, bem como delineamentos que requerem vários dias de comparecimento ao laboratório e campo de avaliação, faz-se necessário compreender as respostas fisiológicas associadas ao desempenho.

Os índices fisiológicos apontados na literatura que mais se associam com desempenho são: o consumo máximo de oxigênio $\left(\mathrm{VO}_{2} \max \right)$; a intensidade do esforço correspondente ao consumo máximo de oxigênio $\left(\mathrm{iVO}_{2} \mathrm{max}\right)$, a economia de movimento (EM) e limiar anaeróbio mensurado pelas respostas ventilatórias (LV) ou lactacidêmicas durante exercícios submáximos ${ }^{1-4}$.

Nesse sentido, alguns estudos demonstram que esses índices fisiológicos são frequentemente utilizados para a predição de desempenho ${ }^{5-7}$, na qual a distância ou o tempo da prova pode ser determinante para a performance do atleta, ou seja, a intensidade do exercício pode influenciar nesta relação ${ }^{6-8}$.

Caputo et al. ${ }^{9}$ demonstraram que $\mathrm{o} \mathrm{VO}_{2} \max , \mathrm{iVO}_{2} \max$ e limiar anaeróbio predizem o desempenho de ciclistas em prova de $4 \mathrm{~km}$ contra relógio $(\mathrm{r}=0,93,0,98,0,92)$ em ciclistas competitivos. Corroborando os achados, Amann et al. ${ }^{10}$, demonstraram que a utilização destes índices fisiológicos podem predizer o desempenho de ciclistas experientes em provas contra relógio (CR) de 40km $\left(\mathrm{VE} / \mathrm{VO}_{2} r=0.80 ; \mathrm{V}\right.$-slope $r=0,79, \mathrm{p}<0,001 ; \mathrm{RER}_{0.95}$ $\left.r=0.73 ; \operatorname{RER}_{1.0} r=0,75, \mathrm{p}<0,01\right)$.

A grande maioria dos estudos envolvendo avaliação de ciclistas empregam cicloergômetros, que apesar da semelhança com a bicicleta e da fina sensibilidade em mensurar o trabalho realizado, não conseguem reproduzir a íntima relação existente entre o atleta, as dimensões da bicicleta e as peças que a compõem ${ }^{11}$. Além disso, é clara e unânime a preferência de ciclistas em realizar procedimentos de avaliação em campo, justamente pelo emprego da própria bicicleta utilizada em treinamento e competição, entretanto, a avaliação em campo torna-se depende de equipamentos específicos e de alto custo para se controlar variáveis fisiológicas ${ }^{11}$.

Dessa maneira, são necessários estudos que possibilitem verificar, nessa população específica e através da própria bicicleta de treinamento e competição, a existência de associação de índices fisiológicos de avaliação aeróbia com o desempenho em provas contra relógio (CR) de curta e média duração. Portanto, o objetivo do presente estudo foi determinar e relacionar diferentes índices fisiológicos de avaliação aeróbia com o desempenho em provas (CR) de $4 \mathrm{~km}$ e $20 \mathrm{~km}$ em ciclistas de alto nível. Como hipótese, uma relação significante entre os parâmetros determinados no teste progressivo com o desempenho nas provas contra relógio é esperada. 


\section{PROCEDIMENTOS METODOLÓGICOS}

\section{Amostra}

A amostra foi composta por 14 ciclistas profissionais de elite nacional do sexo masculino, pertencentes a uma equipe do estado de São Paulo - Brasil, com idade 28,5 $\pm 4,7$ anos, todos com, no mínimo, cinco anos de treinamento, volume de treino $480,6 \pm 30,2 \mathrm{~km} \cdot \mathrm{semana}^{-1}$ e com resultados expressivos em competições nacionais e internacionais, sendo que entre os voluntários para o estudo havia o campeão Pan-americano de velocidade, o campeão brasileiro de Estrada, campeão da Prova 9 de Julho (mais tradicional competição de ciclismo nacional) e campeão brasileiro de contra relógio. Antes de realizar qualquer procedimento, os voluntários foram informados sobre a natureza dos procedimentos, assinaram o Termo de Consentimento Livre e Esclarecido e realizaram uma familiarização com os equipamentos utilizados para a determinação dos índices fisiológicos em laboratório, sendo o estudo previamente aprovado pelo comitê de ética da Faculdade de Ciências e Tecnologia - UNESP - Campus de Presidente Prudente (48/2010).

\section{Coleta de dados}

As avaliações foram realizadas ao longo de três dias distintos, com intervalo de $24 \mathrm{~h}$ entre elas. Todos os voluntários foram instruídos a comparecer aos testes hidratados, a não ingerir bebida cafeinada e alcoólica $24 \mathrm{~h}$ antes das avaliações. No primeiro dia, foram realizadas as avaliações de laboratório, composta de medidas antropométricas e realizado o teste incremental para obtenção dos índices fisiológicos aeróbios. No segundo e terceiro dia, foram realizadas as provas de campo CR de $4 \mathrm{~km}$ e $20 \mathrm{~km}$, sendo todas as avaliações em ordem randômica.

\section{Testes Laboratoriais}

Com a intenção de respeitar os princípios de especificidade e a íntima relação entre as dimensões corporais do ciclista com a bicicleta e seus componentes, os índices fisiológicos foram obtidos por meio de teste continuo e progressivo realizado em laboratório com as próprias bicicletas de treinamento e competição acopladas a um simulador de ciclismo (Cateye CS-1000"). A potência aeróbia máxima $\left(\mathrm{VO}_{2} \mathrm{max}\right)$ foi obtida através do método direto por Software Aerograph 4.3 (AeroSport Inc., Michigan - USA'), analisador de gases (modelo $\mathrm{VO} 2000^{\circ}$ ). O protocolo consistia em um aquecimento de $5 \mathrm{~min}$ a 125 watts de potência e ao final do aquecimento, iniciava-se o teste progressivo com 150 watts de potência e incremento de carga de 25 watts a cada minuto.

\section{Determinação do V0 ${ }_{2}$ max, iV0 2 max e LV}

O teste progressivo foi realizado até a exaustão voluntária do avaliado e o $\mathrm{VO}_{2} \max$ foi determinado através de dois ou mais critérios: coeficiente respiratório $(\mathrm{RQ}) \geq 1,1$, frequência cardíaca próxima da máxima prevista para a idade (220-idade) e/ou existência de platô. $\mathrm{A} \mathrm{iVO}_{2} \max$ foi determinada usando a equação de Kuipers et al. ${ }^{12}$ : 
Equação:

iVO $\max =($ Watts do estágio completo $)+($ tempo do estágio incompleto $/$ tempo total do estágio)* (carga incremental de cada estágio).

Os limiares ventilatórios 1 e 2 (LV1 e LV2) foram determinados, utilizando o método dos equivalentes ventilatórios de $\mathrm{O}_{2}$ e $\mathrm{CO}_{2}$. Assim, LV1 foi determinado pelo aumento do $\mathrm{VE} / \mathrm{VO}_{2}$ sem aumento do $\mathrm{VE} / \mathrm{VCO}_{2}$, de acordo com Caiozzo et al. ${ }^{13}$. Enquanto o LV2 ou ponto de compensação respiratória foi determinado pelo aumento da relação $\mathrm{VE} / \mathrm{VCO}_{2}$ (figura 1). A moda entre três avaliadores conhecedores dos fenômenos foi utilizada para melhor confiabilidade dos resultados ${ }^{14}$.

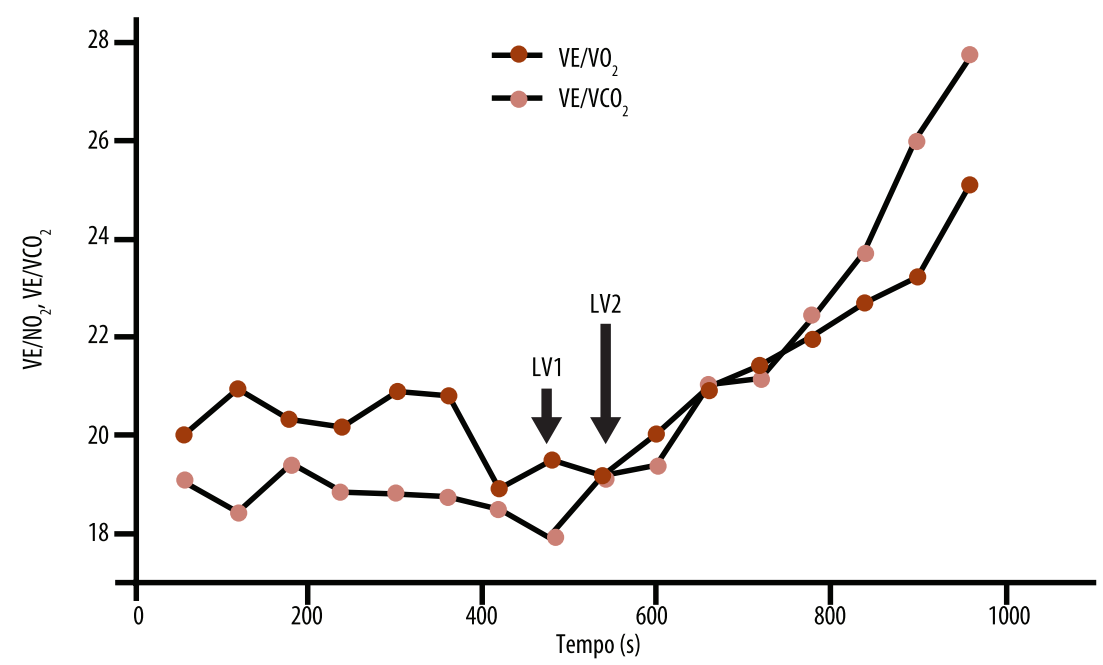

Figura 1. Identificação dos limiares ventilatórios (LV1 e LV2) de acordo com VE/VO 2 e VE/VCO.

\section{Determinação da EM}

A EM foi determinada a partir da relação $\mathrm{VO}_{2} \mathrm{x}$ tempo plotados graficamente abaixo do LV2, onde a EM corresponde ao coeficiente angular da equação: $y=a x+b$; sendo quanto menor o $\mathrm{VO}_{2}$, maior a $\mathrm{EM}$ (figura 2). A frequência cardíaca foi monitorada por meio de um Frequencímetro Polar modelo S-810i, registrada para análise ao final de cada estágio.



Figura 2. Determinação da economia de movimento. 


\section{Determinação do desempenho nas distâncias de 4 e 20 km Contra Relógio}

Os testes de campo foram realizados em uma pista de concreto, totalmente plana de 400 metros, sendo que a angulação das curvas permitia o desenvolvimento de velocidade acima de $47 \mathrm{~km} \cdot \mathrm{h}^{-1}$ sem que o atleta precisasse parar de pedalar. As avaliações iniciaram no período da manhã, com uma temperatura ambiente variando entre $27 \mathrm{e} 30^{\circ} \mathrm{C}$, umidade relativa de ar de $55 \%$ e velocidade do vento variando entre $1,4 \mathrm{e} 2,8 \mathrm{~m} \cdot \mathrm{s}^{-1}$. Após aquecimento de 10 min em intensidade livre (foi indicado que se desenvolvesse o mesmo padrão adotado em aquecimento para competição), os sujeitos pedalavam mais uma volta para atingir a velocidade necessária (saída lançada) para realização dos testes máximos CR nas distâncias de 4 e 20km sem a utilização de vácuo. O tempo total de cada distância foi registrado por meio de um cronômetro manual. Todos os voluntários eram experientes com este modelo de prova, na qual a velocidade é a máxima possível para a distância. Cada atleta utilizou sua própria bicicleta de competição.

\section{Análise estatística}

Após a verificação da distribuição dos dados através do teste de Shapiro-Wilk, foram calculadas as médias, desvio padrão $( \pm \mathrm{DP})$ e coeficiente de variação $(\mathrm{CV})$ para todas as variáveis. As correlações entre os índices fisiológicos e o desempenho CR foram determinadas por meio do teste de correlação de Pearson, pelo software STATISTIC 7.0. Adotou-se um nível de significância inferior a 5\%.

\section{RESULTADOS}

Os valores médios \pm desvio padrão $( \pm \mathrm{DP})$, coeficiente de variação $(\mathrm{CV})$, mínimo e máximo das variáveis fisiológicas avaliadas: $\mathrm{VO}_{2} \max$, frequência cardíaca máxima (FCmax), iVO 2 max, EM, LV1 e LV2, frequência cardíaca correspondente ao LV1, LV2 e percentual relativo ao $\mathrm{VO}_{2} \max \left(\% \mathrm{VO}_{2} \max \right)$ de LV1 e LV2 dos ciclistas estão expressos na tabela 1. A tabela 2 mostra os valores médios \pm DP e CV do desempenho CR de 4 e $20 \mathrm{~km}$. Os valores de correlação entre os índices fisiológicos aeróbios e o tempo de prova CR de $4 \mathrm{~km}$ e $20 \mathrm{~km}$, não foram significantes (Tabela 3).

Tabela 1. Valores médios $\pm D P, C V$, mínimo e máximo das variáveis fisiológicas avaliadas ( $n=14$ ).

\begin{tabular}{|c|c|c|c|}
\hline Variáveis & Médias $\pm \mathrm{DP}$ & CV (\%) & Variação Mínimo - Máximo \\
\hline $\mathrm{VO}_{2 \max }\left(\mathrm{I} \cdot \mathrm{min}^{-1}\right)$ & $4,5 \pm 0,7$ & 16,1 & $3,1-6,0$ \\
\hline $\mathrm{VO}_{2 \max }\left(\mathrm{ml} \cdot \mathrm{kg}^{-1} \cdot \mathrm{min}^{-1}\right)$ & $62,2 \pm 8,2$ & 13,3 & $45,6-75,8$ \\
\hline $\mathrm{FC}_{\max }\left(\mathrm{bpm}^{-1}\right)$ & $184,7 \pm 5,7$ & 3,1 & $173-191$ \\
\hline $\mathrm{EM}\left(\mathrm{ml} \cdot \mathrm{kg}^{-1} \cdot \mathrm{min}^{-1} \cdot \mathrm{w}^{-1}\right)$ & $0,1166 \pm 0,0362$ & 31,0 & $0,0527-0,1957$ \\
\hline $\mathrm{iVO}_{2 \max }(\mathrm{W})$ & $500,8 \pm 58,6$ & 11,7 & $401,6-593,7$ \\
\hline LV1 (W) & $348,2 \pm 43,26$ & 12,4 & $275-425$ \\
\hline LV1 $\left(b_{p m}^{-1}\right)$ & $159,7 \pm 9,19$ & 5,8 & $144-171$ \\
\hline LV1 $\left(\% \mathrm{VO}_{2 \max }\right)$ & $69,6 \pm 5,2$ & 7,5 & $61,2-77,2$ \\
\hline LV2 (W) & $417,8 \pm 60,79$ & 14,5 & $325-550$ \\
\hline LV2 $\left(\mathrm{bpm}^{-1}\right)$ & $172,7 \pm 8,95$ & 5,2 & $160-188$ \\
\hline LV2 $\left(\% \mathrm{VO}_{2 \max }\right)$ & $83,4 \pm 6,7$ & 8,1 & $74,8-95,1$ \\
\hline
\end{tabular}

$\mathrm{V}_{{ }_{2 \max }}=$ Consumo Máximo de 0xigênio; $\mathrm{FC}_{\max }=$ Frequência Cardíaca Máxima; $\mathrm{EM}=$ Economia de Movimento; $\mathrm{iVO}_{2 \max }^{2_{\max }}=$ Intensidade de Exercício Correspondente ao Consumo Máximo de 0xigênio; LV1 = Limiar Ventilatório 1; LV2 $\stackrel{2 m a x}{=}$ Limiar Ventilatório 2. 
Tabela 2. Valores médios $\pm D P$ e CV do desempenho CR de 4km e $20 \mathrm{~km}$.

\begin{tabular}{lcc}
\hline & Média \pm DP & $C V(\%)$ \\
\hline $4 \mathrm{~km}(\mathrm{~s})$ & $332,3 \pm 12,4$ & 3,7 \\
$20 \mathrm{~km}(\mathrm{~s})$ & $1801,5 \pm 86,4$ & 4,8 \\
\hline
\end{tabular}

CV = Coeficiente de Variação.

Tabela 3. Correlação entre índices fisiológicos aeróbios e o desempenho CR nas distâncias de 4km e 20km.

\begin{tabular}{|c|c|c|c|c|c|c|}
\hline Variáveis & $\mathrm{VO}_{2} \max \left(1 \cdot \min ^{-1}\right)$ & $\begin{array}{l}\mathrm{VO}_{2} \max \\
\left(\mathrm{ml}^{\prime} \cdot \mathrm{kg}^{-1} \cdot \mathrm{min}^{-1}\right)\end{array}$ & $\mathrm{iVO}_{2} \max (\mathrm{w})$ & $\begin{array}{l}\mathrm{EM} \\
\left(\mathrm{ml} \cdot \mathrm{kg}^{-1} \cdot \mathrm{min}^{-1} \cdot \mathrm{w}^{-1}\right)\end{array}$ & $\begin{array}{l}\text { LV1 } \\
(w)\end{array}$ & $\begin{array}{l}\text { LV2 } \\
\text { (w) }\end{array}$ \\
\hline CR 4km (s) & $r=0,38 p=0,17$ & $\begin{array}{l}r=0,16 \\
p=0,57\end{array}$ & $r=-0,33 p=0,23$ & $\begin{array}{l}r=0,20 \\
p=0,47\end{array}$ & $r=-0,50 p=0,06$ & $r=-0,20 p=0,48$ \\
\hline CR 20km (s) & $r=0,24 p=0,41$ & $\begin{array}{l}r=0,01 \\
p=0,96\end{array}$ & $r=-0,13 p=0,64$ & $\begin{array}{l}r=-0,12 \\
p=0,69\end{array}$ & $r=-0,48 p=0,09$ & $r=-0,19 p=0,52$ \\
\hline
\end{tabular}

$\mathrm{VO}_{2 \max }=$ Consumo Máximo de 0xigênio; iV0 ${ }_{2 \max }=$ Intensidade de Exercício Correspondente ao Consumo; Máximo de 0xigênio; EM = Economia de Movimento; LV1 = Limiar Ventilatório 1; LV2 = Limiar Ventilatório 2; $C R=$ Provas Contra Relógio.

\section{DISCUSSÃO}

O objetivo do presente estudo foi determinar e relacionar diferentes índices fisiológicos de avaliação aeróbia $\left(\mathrm{VO}_{2} \max , \mathrm{iVO}_{2} \max , \mathrm{EM}, \mathrm{LV} 1\right.$ e LV2) determinados em teste laboratoriais, com o desempenho em provas CR de $4 \mathrm{~km}$ e $20 \mathrm{~km}$ em ciclistas de elite.

O principal achado do estudo foi a não correlação significante do ponto de vista estatístico entre os índices fisiológicos aeróbios e o desempenho dos atletas, sugerindo que tais variáveis, para estes sujeitos, não são sensíveis para predizer o desempenho nas respectivas distâncias, discordando da hipótese apresentada no estudo.

$\mathrm{O}$ valor de $\mathrm{VO}_{2}$ max do presente estudo é superior quando comparado $\left(62,23 \pm 8,28 \mathrm{ml} \cdot \mathrm{kg}^{-1} \cdot \mathrm{min}^{-1}\right)$ ao de atletas nacionais apresentados por Caputo et al. ${ }^{9}\left(58,80 \pm 8,4 \mathrm{ml} \cdot \mathrm{kg}^{-1} \cdot \mathrm{min}^{-1}\right)$, Okano et al. ${ }^{15}\left(57,50 \pm 4,22 \mathrm{ml} \cdot \mathrm{kg}^{-1} \cdot \mathrm{min}^{-1}\right)$ e Diefenthaler et al. ${ }^{8}\left(57,72 \pm 3,92 \mathrm{ml} \cdot \mathrm{kg}^{-1} \cdot \mathrm{min}^{-1}\right)$. Segundo Wilmore e Costill ${ }^{16}$, os valores absolutos de $\mathrm{VO}_{2}$ max podem se correlacionar melhor com o desempenho do que os valores relativos à massa corporal em ciclistas, pois durante a competição, a interferência da força da gravidade é menor quando comparada à corrida. Entretanto, não foram verificadas essas correlações na presente investigação. Esses resultados reforçam os achados de Coyle et al. ${ }^{17}$ e Impellizzeri et al. ${ }^{7}$ que demonstraram que indivíduos bem treinados ou atletas de elite que apresentam valores elevados de $\mathrm{VO}_{2} \max$, não apresentam correlação com o desempenho. Este índice fisiológico está relacionado a fatores centrais (débito cardíaco) e parece sofrer adaptações moderadas ou, ainda, não se modificar em resposta ao treinamento ${ }^{19,20}$. Portanto, os atletas podem continuar sofrendo adaptações metabólicas (resposta do lactato ao exercício) e neuromusculares (EM) positivas, sem alteração no débito cardíaco. Sendo assim, melhoram suas performances sem mudanças no $\mathrm{VO}_{2} \max ^{21}$.

Em nosso estudo, a EM não se correlacionou com o desempenho dos atletas (EM $v s 4 \mathrm{~km} r=0,20, p=0,471$; EM $v s 20 \mathrm{~km} r=-0,12, p=0,691$ ), além disso, apresentou elevada variação interindividual $(C V=31,07 \%)$ e eleva- 
da associação com $\mathrm{VO}_{2} \max (r=0,77, p<0,05)$ corroborando o estudo de Caputo et al. ${ }^{9}$. A EM é pouco sensível na predição de desempenho, e sua variação $(15 \%)^{9}$ influencia a $\mathrm{iVO}_{2}$ max mesmo em grupos de atletas com valores similares de $\mathrm{VO}_{2} \max ^{20}$.

$\mathrm{A} \mathrm{iVO}_{2}$ max também não apresentou correlação com o desempenho (4 $\mathrm{km} r=-0,33, p=0,23920 \mathrm{~km} r=-0,13, p=0,649)$, ao contrário dos achados de alguns autores ${ }^{9,22}$ que consideram a $\mathrm{iVO}_{2}$ max capaz de predizer desempenho em provas de curta duração (3-5min), tendo em vista a grande contribuição aeróbia $(>84 \%)$ em provas de $1500 \mathrm{~m}$ em corredores ${ }^{18}$. Entretanto, devemos enfatizar a contribuição relativa dos sistemas energéticos aeróbio e anaeróbio nas distâncias das provas que foram analisadas. Denadai et al. ${ }^{18}$ sugerem, assim, que a avaliação visando a predição de desempenho e/ou o acompanhamento dos efeitos do treinamento de atletas de meia-distância incluam a determinação do tempo limite (Tlim) ou do máximo déficit acumulado de oxigênio (MAOD) proposto por Medbo et al. ${ }^{23}$ que são parâmetros de capacidade anaeróbia e podem explicar a não correlação da $\mathrm{iVO}_{2}$ max com o desempenho dos ciclistas.

Para provas de média e longa duração ( $\geq 25 \mathrm{~min}$ ), como no caso do teste de $20 \mathrm{~km}(1801,5 \pm 86,4 \mathrm{~s})$, a capacidade aeróbia, representada pelos limiares ventilatórios (LV1: 348,21 \pm 43,26 w e LV2: 417,86 \pm 60,79 w) tem papel decisivo no desempenho ${ }^{10}$. Entretanto, também não foi encontrada correlação entre estes índices fisiológicos e o desempenho no teste de $20 \mathrm{~km}$. É possível que estes resultados se relacionem ao método de determinação do limiar anaeróbio empregado em nosso experimento, realizado através de variáveis ventilatórias e não pelo padrão ouro de avaliação da capacidade aeróbia (máxima fase estável de lactato) ${ }^{3}$. Entretanto, Amann et al. ${ }^{10}$ evidenciaram que o LV é uma variável mais sensível que o limiar determinado por variável lactacidêmica para a predição do desempenho em prova de $40 \mathrm{~km}$ CR. Os autores apontam que a ventilação pulmonar pode ser um indicador da concentração de íons $\mathrm{H}^{+}$mais sensível que a concentração de lactato.

Para Caputo et al. ${ }^{9}$, as associações que determinados índices fisiológicos têm com o desempenho podem sofrer influência do tipo de exercício, da duração da prova, estado de treinamento e/ou baixa variação de desempenho interindividual (homogeneidade dos atletas). O coeficiente de variação encontrado tanto no desempenho de $4 \mathrm{~km} \mathrm{CR}(3,7 \%)$ quanto de $20 \mathrm{~km}$ CR $(4,8 \%)$ demonstram a baixa variação interindividual dos ciclistas. Outra hipótese é o fato dos índices fisiológicos terem sido determinados em testes laboratoriais, uma vez que estudos demonstram que avaliações em campo em ciclistas apresentam maior correlação com o desempenho do que testes laboratoriais ${ }^{11,24}$, apesar de utilizarmos em nosso experimento o simulador de ciclismo, que permite que o atleta realize o procedimento de avaliação com sua própria bicicleta.

No entanto, a não correlação entre os índices determinados neste estudo e o desempenho dos ciclistas, direcionam a formulação de algumas hipóteses como: a participação das vias anaeróbias na performance, que 
embora também não tenha sido verificada, pode ter contribuído para o desempenho, especialmente na prova CR de $4 \mathrm{~km}$, que apresentou tempo de esforço bem abaixo de $30 \mathrm{~min}$. Além disso, pelo fato dos esforços de $4 \mathrm{e}$ $20 \mathrm{~km}$ terem apresentado tempo médio de $5 \mathrm{~min} 32 \mathrm{seg}$ e $31 \mathrm{~min} 41 \mathrm{seg}$ (tabela 2) e sido realizados durante em esforço máximo, pode-se afirmar que a velocidade selecionada pelos participantes foi acima do limiar anaeróbio ${ }^{25}$, fato comprovado pela concentração de lactato após os esforços de 4 e $20 \mathrm{~km}$ $\left(15,52 \pm 2,50\right.$ e $8,45 \pm 3,05 \mathrm{mmol} \cdot \mathrm{l}^{-1}$, respectivamente); uso de testes laboratoriais para a determinação das variáveis fisiológicas, eliminando assim, fatores externos; os atletas serem de alto nível e terem baixo coeficiente de variação no desempenho CR nas distâncias de 4 e 20km e, segundo Mujika e Padilla ${ }^{11}$, os valores referentes aos índices fisiológicos quando expressos em valores relativos à massa corporal magra total do corpo, apresentam maior correlação com desempenho, dependendo da especialidade do atleta, quando comparado a valores absolutos. Dessa maneira, os índices fisiológicos aeróbios não foram suficientemente sensíveis para predizer o desempenho dos ciclistas.

As limitações do presente estudo foram: (i) a avaliação laboratorial pode não apresentar validade ecológica para provas em campo (devido à condições climáticas); (ii) a influência da composição corporal na performance em ciclistas de elite; e (iii) a não avaliação da aptidão anaeróbia dos atletas do presente estudo.

\section{CONCLUSÃO}

Portanto, pode-se concluir que os índices fisiológicos aeróbios determinados em laboratório não se correlacionaram com o desempenho em provas de campo contra relógio de $4 \mathrm{~km}$ e $20 \mathrm{~km}$ e, assim, não foram capazes de predizer desempenho para grupo de ciclistas profissionais de elite em provas de CR. Os resultados do presente estudo evidenciam que atletas e treinadores devem lançar mão de avaliações mais específicas (em campo) a fim de conseguir predizer com maior segurança o desempenho de ciclistas de elite.

\section{REFERÊNCIAS BIBLIOGRÁFICAS}

1. Jacobs RA, Rasmussen P, Siebnmann C, Diaz V, Gassmann M, Pesta D, et al. Determinants of time trial performance and maximal incremental exercise in highly trained endurance athletes. J Appl Physiol 2011;111(5):1422-30.

2. Caputo F, De Oliveira MFM, Greco CC, Denadai BS. Exercícios aeróbios: aspectos bioenergéticos, ajustes fisiológicos, fadiga e índices de desempenho. Rev Bras Cineantropom Desempenho Hum 2009;11(1):94-102.

3. Figueira TR, Denadai BS. Relações entre o limiar anaeróbio, limiar anaeróbio individual e máxima fase estável de lactato em ciclistas. Rev Bras Ciênc Mov 2004;12(1):91-5.

4. Fabre N, Passelergue P, Bouvard M, Perrey, Perrey S. Comparison of heart rate deflection and ventilatory threshold during a Field cross-crountry roller-sking test. J Strength Cond Res 2008;22(6):1977-84.

5. Lucia A, Hoyos J, Pérez M, Santalla A, Earnest CP, Chicharro JL. Which laboratory variable is related with time trial performance time in the Tour de France?. Br J Sports Med 2004;38(5):636-40. 
6. Faria EW, Parker DL, Faria IE. The Science of Cycling Physiology and Training Part 1. Sports Medicine 2005;35(4):285-312.

7. Impellizzeri FM, Marcora SM, Rampinini E, Mognoni P, Sassi A. Correlations between physiological variables and performance in high level cross country off road cyclists. Br J Sports Med 2005;39(10):747-51.

8. Diefenthaeler F, Candotti CT, Ribeiro J, Oliveira AR. Comparação de respostas fisiológicas absolutas e relativas entre ciclistas e triatletas. Rev Bras Med Esporte 2007;13(3):205-8.

9. Caputo F, Lucas RD, Mancini E, Denadai BS. Comparação de diferentes índices obtidos em testes de campo para predição da performance aeróbia de curta duração no ciclismo. Rev Bras Ciênc Mov 2001;9(4):13-7.

10. Amann M, Subudhi AW, Foster C. Predictive validity of ventilatory an lactate thresholds for cyclisng time trial performance. Scand J Med Sci Sports 2006;16(1):27-34.

11. Mujika I, Padilla S. Adaptations to training in endurance cyclists. Implications for the performance. Sports Med 2001;31(7):511-20.

12. Kuipers H, Verstappen FT, Keizer HA. Variability of aerobic performance in the laboratory and its physiologic correlates. Int J Sports Med 1985;6(4):197-201.

13. Caiozzo VJ, Davis JA, Ellis JF, Azus JL, Vandagriff R, Prietto CA, McMaster WC. A comparison of gas exchange indices used to detect the anaerobic threshold. J Appl Physiol 1982;53(5):1184-9.

14. Pires FO, Silva AEL, De Oliveira FR. Diferenças entre variáveis de identificação dos limiares ventilatórios. Rev Bras Cineantropom Desempenho Hum 2005;7(2):20-8.

15. Okano AH, Altimari LR, Simões HG, De Moraes AC, Nakamura FY, Cyrino ES, Burini EC. Comparação entre limar anaeróbio determinado por variáveis ventilatórias e pela resposta do lactato sanguíneo em ciclistas. Rev Bras Med Esporte 2006;12(1):39-44.

16. Wilmore JH, Costill DL. Physiology of Sports and Exercise. Champaingn: Human Kinects, 1994.

17. Coyle EF, Feltner ME, Kautz SA, Hamilton MT, Montain SJ, Baylor AM, et al. Physiological and biomechanical factors associated with elite endurance cycling performance. Med Sci Sports Exerc 1991;23(1):93-107.

18. Denadai BS, Ortiz MJ, de Mello MT. Índices fisiológicos associados com a "performance" aeróbia em corredores de "endurance" efeitos da duração da prova. Rev Bras Med Esporte 2004;10(5):401-4.

19. Saltin B, Strange S. Maximal oxygen uptake: "old" and "new" arguments for a cardiovascular limitation. Med Sci Sports Exerc 1992;24(1):30-7.

20. Ortiz MJ, Denadai BS, Stella S, de Mello MT. Efeitos do treinamento aeróbio de alta intensidade sobre a economia de corrida em atletas de endurance. Rev Bras Ciênc Mov 2003;11(3):53-6.

21. Howley JA, Stepto NK. Adaptations to training in endurance cyclists. Implications for performance. Sports Med 2001;31(7):511-20.

22. Billat V, Beillot J, Jan J, Rochcongar P, Carre F. Gender effect on the relationship of time limit at 100\%O2max with other bioenergetic characteristics. Med Sci Sports Exerc 1996;28(8):1049-55.

23. Medbo JI, Mohn AC, Tabata I, Bahr R, Vaage O, Sejersted OM. Anaerobic capacity determined by maximal accumulated O2 deficit. J Appl Physiol 1988;64(1):50-60.

24. Balikian PJ, Denadai BS. Aplicações do limiar anaeróbio determinado em teste de campo para o ciclismo: comparação com valores obtidos em laboratório. Motriz 1996;2(1):26-32.

25. Kenefick RW, Mattern CO, Mahood NV, Quinn TJ. Physiological variables at lactate threshold under represent cycling time-trial intensity. J Sports Med Phys Fitness 2002; 42(4):396-402.

\section{Endereço para correspondência}

Eduardo Bernardo Sangali Rua Barão de Cotegipe, n¹280, Vila Tibério.

CEP: 14050-420 - Ribeirão Preto, SP. Brasil

E-mail:du_bs1@hotmail.com 